\title{
Vascular Risk Factors, Imaging, and Outcomes in Transient Ischemic Attack/Ischemic Stroke Patients with Neuroimaging Evidence of Probable/Possible Cerebral Amyloid Angiopathy
}

\author{
Qihui Zhang, ${ }^{1,2}$ Anxin Wang, ${ }^{1,2}$ Xia Meng, ${ }^{2}$ Xiaoling Liao, ${ }^{1,2}$ Yijun Zhang,,2 \\ Huiqing Hou, ${ }^{1,2,3}$ Lijuan Niu, ${ }^{2}$ Ruiping $\mathrm{Li}^{4}{ }^{4}$ Wenjuan Guo, ${ }^{4}$ and Yongjun Wang $\mathbb{D}^{1,2}$ \\ ${ }^{1}$ Department of Neurology, Beijing Tiantan Hospital, Capital Medical University, Beijing, China \\ ${ }^{2}$ China National Clinical Research Center for Neurological Diseases, Beijing, China \\ ${ }^{3}$ Department of Neurology, The Second Hospital of Hebei Medical University, Shijiazhuang, China \\ ${ }^{4}$ Beijing University of Chinese Medicine, Beijing, China
}

Correspondence should be addressed to Yongjun Wang; yongjunwang@ncrcnd.org.cn

Received 13 March 2021; Revised 11 April 2021; Accepted 17 April 2021; Published 27 April 2021

Academic Editor: Yue Liu

Copyright (c) 2021 Qihui Zhang et al. This is an open access article distributed under the Creative Commons Attribution License, which permits unrestricted use, distribution, and reproduction in any medium, provided the original work is properly cited.

\begin{abstract}
Background. In TIA/ischemic stroke patients, the clinical significance of lobar microbleeds potentially indicating cerebral amyloid angiopathy (CAA) is unknown. We assessed vascular risk factors and outcomes, including cognition, in TIA/ischemic stroke patients with neuroimaging evidence of probable/possible CAA. Methods. This prospective cohort was conducted from August 2015 and January 2018 at 40 centers. 2625 participants were collected. Eligible participants were aged at least 55 years. Montreal Cognitive Assessment (MoCA) score is less than or equal to 26. A total of 1620 patients were included. 1604 (99.0\%) and 1582 (97.7\%) participants are followed up at 3 and 12 months. The primary outcomes were death or disability (mRS score, 3-6) and Montreal Cognitive Assessment (MoCA) at 3 months and 12 months. Demographic and vascular risk factors were measured at baseline (smoking, alcohol, diabetes, atrial fibrillation, hypertension, hypercholesterolemia, coronary artery disease, ischemic stroke, and transient ischemic attack). Blood samples were collected within 24 hours of admission. MRI was recommended for all patients. MoCA score was evaluated at baseline and follow-up. Results. In total, 291/1620 patients with ischemic stroke/TIA (32.7\% female and mean age, 67.8 years) had neuroimaging evidence of probable/possible CAA. Higher age, history of hypertension, atrial fibrillation, ischemic stroke, alcohol, and high glucose at the admission were more common in the patients. Mean MoCA changed from 21.4 at 3 months (SD 5.2) to 22.3 at 12 months (SD 4.7), difference 0.3 (SD 3.8). At the 3-month and 12-month follow-up, there were significant differences in age, education level, and sex among different cognitive groups. Higher age, lower education (less than high school), and female sex were the predictors of changing in MoCA score from 3 months to 12 months. Moreover, age (more than 66 years) and education (less than high school) are strongly associated with MoCA at 3- and 12-month follow-up. 30 of 286 (10.5\%) and 37 of 281 (13.2\%) patients had poor outcome of death or disability (modified Rankin Scale score, 3-6) at follow-up 3 and 12 months. Cortical superficial siderosis (cSS) was associated with higher mRS at follow-up. cSS status, cSS count 1-2, cSS strictly lobar, and strictly deep might be the risks of outcomes in adjusted analyses. Conclusion. This study suggested that an increasing number of vascular risk factors and imaging markers were significantly associated with outcomes of TIA/ischemic stroke patients with CAA pattern. Male, young patients with high education should get better cognitive recovery.
\end{abstract}

\section{Introduction}

Cognitive impairment and degree of global disability after TIA or ischemic stroke is the most common outcome in some clinical trials. Cerebral amyloid angiopathy (CAA) is a small vessel disease of the brain, which leads to cognitive impairments in elderly people [1-3]. The impact of cerebral amyloid angiopathy to poststroke cognitive impairment and global disability in TIA/ischemic stroke patients is indeterminate. 
TABLE 1: Characteristics of TIA/ischemic stroke patients with neuroimaging evidence of probable/possible CAA pattern.

\begin{tabular}{|c|c|c|c|c|}
\hline Characteristic & All patients $(n=1620)$ & CAA pattern $(n=291)$ & Non-CAA pattern $(n=1329)$ & $p$ value \\
\hline Age, mean (SD), y & $66.5(7.6)$ & $67.8(7.7)$ & $66.2(7.5)$ & 0.001 \\
\hline Sex (female), No., \% & $490(30.3 \%)$ & $95(32.7 \%)$ & $395(29.7)$ & 0.33 \\
\hline BMI, mean (SD) & $24.8(3.2)$ & $24.9(3.2)$ & $24.8(3.2)$ & 0.52 \\
\hline HTN No., \% & $1045(64.5 \%)$ & $212(72.9 \%)$ & $833(62.7 \%)$ & 0.001 \\
\hline DM No., \% & $387(23.9 \%)$ & $65(22.3 \%)$ & $322(24.2 \%)$ & 0.49 \\
\hline AF No., \% & $111(6.9 \%)$ & $29(10.0 \%)$ & $82(6.2 \%)$ & 0.02 \\
\hline Hyperlipidemia No., \% & $148(9.1 \%)$ & $23(7.9 \%)$ & $125(9.4 \%)$ & 0.42 \\
\hline Coronary artery disease No., $\%$ & $11(0.7 \%)$ & $4(1.4 \%)$ & $7(0.5 \%)$ & 0.11 \\
\hline Prior ischemic stroke No., \% & $391(24.1 \%)$ & $91(31.3 \%)$ & $300(22.6 \%)$ & 0.002 \\
\hline Prior TIA No., \% & $47(2.9 \%)$ & $10(3.4 \%)$ & $37(2.8 \%)$ & 0.83 \\
\hline Current smoking No., \% & $505(31.2 \%)$ & $86(29.6 \%)$ & $419(31.5 \%)$ & 0.51 \\
\hline Alcohol No., \% & $239(14.8 \%)$ & $32(11.0 \%)$ & $207(15.6 \%)$ & 0.046 \\
\hline \multicolumn{5}{|l|}{ Education level No., \% } \\
\hline >high school & $118(7.3 \%)$ & $22(7.6 \%)$ & $96(7.2 \%)$ & \multirow{3}{*}{0.56} \\
\hline High school & $344(21.2 \%)$ & $55(18.9 \%)$ & $289(21.8 \%)$ & \\
\hline$<$ high school & $1158(71.5 \%)$ & $241(73.5 \%)$ & $944(71.0 \%)$ & \\
\hline Initial mRS, mean (SD) & $1.8(1.3)$ & $1.8(1.3)$ & $1.8(1.3)$ & 0.44 \\
\hline Initial MoCA, mean (SD) & $19.2(5.3)$ & $18.9(5.4)$ & $19.3(5.3)$ & 0.24 \\
\hline Glucose mean (IQR), mg/dL & $6.3(2.4)$ & $6.1(2.4)$ & $6.4(2.4)$ & 0.03 \\
\hline $\mathrm{Hgb} g / \mathrm{L}^{\mathrm{c}}$ & $140.6(16.2)$ & $141.1(16.6)$ & $140.5(16.1)$ & 0.50 \\
\hline Plt $* 10^{9} / \mathrm{L}^{\mathrm{d}}$ & $206.0(56.8)$ & $199.0(58.7)$ & $207.5(56.3)$ & 0.06 \\
\hline $3 \mathrm{~m} \mathrm{MoCA}^{\mathrm{e}}$ & $22.1(5.0)$ & $21.4(5.2)$ & $22.3(4.9)$ & 0.03 \\
\hline $12 \mathrm{~m} \mathrm{MoCA}^{\mathrm{f}}$ & $22.4(4.9)$ & $22.3(4.7)$ & $22.4(5.0)$ & 0.85 \\
\hline
\end{tabular}

Abbreviations: CAA: cerebral amyloid angiopathy; BMI: body mass index; HTN: hypertension; DM: diabetes; AF: atrial fibrillation; TIA: transient ischemic attack; mRS: modified Rankin Scale; MoCA: Montreal Cognitive Assessment; Hgb: hemoglobin; Plt: platelet count. ${ }^{\mathrm{b}}$ Data were missing for 227 patients. ${ }^{c}$ Data were missing for 9 patients. ${ }^{\mathrm{d}}$ Data were missing for 12 patients. ${ }^{\mathrm{e}}$ Data were missing for 233 patients. ${ }^{\mathrm{f}}$ Data were missing for 724 patients.

Vascular risk factors, including smoking, hypertension, diabetes, hyperlipidemia, and atrial fibrillation (AF), are strongly associated with cognitive decline and global disability $[4,5]$. Previous studies have comprehensively presented the influence risk of magnetic resonance imaging (MRI) to CAA. They focused on cerebral microbleeds (CMBs), white matter changes (WMHs), and cortical superficial siderosis (cSS) [6]. These three are often closely related. The consensus regarding neuroimaging marker for CAA is clinically meaningful [7]. The evaluation of the interactive effects of vascular risk and imaging markers is more important to the outcome of neuroimaging evidence of CAA pattern in ischemic intracerebral events (TIA and ischemic stroke).

In this study, we used data from China National Clinical Research Center for Neurological Diseases to mimic the design and to examine the China National Stroke Registry III Cognitive Subgroup (ICONS) participants at follow-up 3 and 12 months, when vascular risks and neuroimaging marker assessment appeared to be the most critical. We investigated cognitive impairment and the degree of global disability in patients with neuroimaging evidence of probable/possible CAA-related ischemic stroke or TIA. We hypothesized that vascular risk factors and imaging profile would be associated with the modified Rankin Scale (mRS) and that cognitive impairments at 3 and 12 months. On the basis of the highlighting the cognitive performance is associ- ated with cerebral small-vessel disease at 12 months [8], we were interested in the factors predicting cognitive impairment in CAA pattern patients in TIA or ischemic stroke and comparing patients who improve between 3 and 12 months with those that do not.

\section{Materials and Methods}

2.1. Study Design. In this prospective study, we used data from the Impairment of Cognition and Sleep (ICONS) after acute ischemic stroke or transient ischemic attack study in Chinese patients conducted from August 2015 to January 2018. In 201 hospitals of the China National Stroke Registry III study, the steering committee of ICONS chose 40 hospitals nationwide to represent the population from each region of east, south, west, north, and center of Mainland China [9]. Consecutive IS or TIA in-hospital patients within 7 days after onset were enrolled in ICONS study. The study followed up 3 and 12 months.

2.2. Participants. In brief, the prospective cohort ICONS study recruited 2625 participants in China. The present analysis included 1620 selected patients, their age was $\geq 55 \mathrm{y}$, and MoCA score is $\leq 26$. All patients tested brain MRI, including T2-weighted, T2*-weighted gradientrecalled echo, and/or susceptibility-weighted imaging and 
fluid-attenuated inversion recovery sequences. 291 patients were clinically diagnosed CAA pattern by the Boston Criteria [10].

2.3. Data Collection. Demographic information and vascular risk factors, imaging, and cognitive tests were prospectively recorded at the time of study enrollment and then followed up for 3 months and 12 months. This has been described previously for the ICONS study.

2.4. Covariates. All 1620 visits include vascular risk factor assessments, defined atrial fibrillation, coronary artery disease, prior ischemic stroke, prior TIA, prior ICH, current smoking, and alcohol. The presence of diabetes (fasting glucose level $\geq 126 \mathrm{mg} / \mathrm{dL}$, nonfasting glucose level $\geq$ $200 \mathrm{mg} / \mathrm{dL}$, self-report of physician-diagnosed diabetes, or use of oral diabetes medications or insulin), hypertension (blood pressure was measured twice in resting state on different days, systolic blood pressure $\geq 140 \mathrm{mmHg}$ and/or diastolic blood pressure $\geq 90 \mathrm{mmHg}$ or physician-diagnosed hypertension and taking antihypertensive drugs), and hyperlipidemia [defined by patient having past history of hyperlipidemia, using a lipid-lowering agent including statins or patient diagnosed hyperlipidemia at admission as having serum cholesterol $>240 \mathrm{mg} / \mathrm{dL}$ or low - density lipoprotein cholesterol (LDL - cholesterol) levels $\geq 130 \mathrm{mg} / \mathrm{dL}$ ] were recorded. For demographic factors, age, sex, and education level (college graduate or professional school, high school, junior school, primary school, and illiteracy) were selfreported. Body mass index (BMI) was calculated as weight in kilograms divided by height in meters squared. Plasma glucose, hemoglobin (Hgb), and platelet count (Plt) were measured. The modified Rankin Scale (mRS) is defined categorically with 7 levels of patient functional independence following a stroke, with scores ranging from 0 (fully independent) to 6 (death) [11]. According to the MoCA score, cognitive function was divided into three groups: normal (more than 26 score), mild (18-26 score), moderate, and severe (no more than 17 score) [12]. MoCA score was evaluated at admission and follow-up 3 and 12 months.

2.5. Imaging Acquisition and Analysis. Brain MRI scans, obtain as research studies at entry. All MRI images were reviewed centrally by two neuroradiologists.

MRI factors included diffusion-weighted imaging hyperintense lesions (DWIHLs), defined as regions of intraparenchymal hyperintensity on diffusion-weighted imaging, with associated hypointensity or matter hyperintensity (WMH) was evaluated visually on fluid-isointensity on apparent diffusion coefficients. White attenuated inversion recovery images using the Fazekas scale.

The number and location of cerebral microbleeds were identified according to the Brian Observer Micro Bleed Scale (BOMBS) on haem-sensitive MRI sequences [13], also known as $\mathrm{T}^{*}$ or gradient echo (GRE). There are 7 locations which should be assessed: contex/gray-white junction, subcortical white matter, basal ganglia grey matter, brainstem, cerebellum, internal/external capsule, and thalamus. The diagram demonstrates in the appropriate row for rating size
TABLE 2: MR imaging findings by status of CAA pattern in TIA/ischemic stroke patients.

\begin{tabular}{|c|c|c|}
\hline Variable & $\begin{array}{l}\text { CAA pattern } \\
(n=291)\end{array}$ & $\begin{array}{c}\text { Without CAA } \\
\text { pattern }(n=1329)\end{array}$ \\
\hline $\begin{array}{l}\text { Fazekas scale score, mean } \\
(\mathrm{IQR})^{\mathrm{a}}\end{array}$ & $3.63(2-5)$ & $2.61(1-4)$ \\
\hline CMB count (No., \%) & $241(82.3 \%)$ & $38(2.9 \%)$ \\
\hline \multicolumn{3}{|l|}{ CMB topography (No., \%) } \\
\hline \multicolumn{3}{|c|}{ Contex/grey-white junction } \\
\hline Number $<5 \mathrm{~mm}$ & $156(53.6 \%)$ & $1(0.1 \%)$ \\
\hline Number 5-10 mm & $14(4.8 \%)$ & $0(0)$ \\
\hline \multicolumn{3}{|l|}{ Subcortical white matter } \\
\hline Number $<5 \mathrm{~mm}$ & $47(16.2 \%)$ & $1(0.1 \%)$ \\
\hline Number 5-10 mm & $2(0.7 \%)$ & $0(0)$ \\
\hline \multicolumn{3}{|c|}{ Internal and external capsule } \\
\hline Number $<5 \mathrm{~mm}$ & $6(2.1 \%)$ & $12(0.9 \%)$ \\
\hline Number $5-10 \mathrm{~mm}$ & $0(0)$ & $0(0)$ \\
\hline \multicolumn{3}{|l|}{ Cerebellum } \\
\hline Number $<5 \mathrm{~mm}$ & $59(20.3 \%)$ & $0(0)$ \\
\hline Number 5-10 mm & $5(1.7 \%)$ & $0(0)$ \\
\hline cSS count (No., \%) & $95(32.7 \%)$ & $42(3.2 \%)$ \\
\hline \multicolumn{3}{|l|}{ cSS topography (No., \%) } \\
\hline Frontal & $10(3.4 \%)$ & $1(0.1 \%)$ \\
\hline Parietal & $14(4.8 \%)$ & $1(0.1 \%)$ \\
\hline Temporal & $37(12.7 \%)$ & $2(0.2 \%)$ \\
\hline Occipital & $13(4.5 \%)$ & $0(0)$ \\
\hline Centrum semiovale & $6(2.1 \%)$ & $0(0)$ \\
\hline Cerebellum & $11(3.8 \%)$ & $0(0)$ \\
\hline
\end{tabular}

Abbreviations: CMB: cerebral microbleed; cSS: cortical superficial siderosis. ${ }^{\mathrm{a}}$ Data were missing for 193 patients.

( $<5 \mathrm{~mm}, 5-10 \mathrm{~mm})$. We followed the methods of Ashkan et al. [14]. The CMB count severity was coded a prior as absent (0 CMBs), mild (1-2 CMBs), moderate (3-10 CMBs), or severe (>10 CMBs) [15]. The CMB topography was coded as strictly lobar (with or without concurrent CMBs), strictly deep (deep and/or CMBs), or mixed (concurrent lobar and deep CMBs). The number and location of cSS were classified as CMBs.

2.6. Statistical Analysis. Patient demographic and MR imaging markers were compared between groups in crosssectional analyses using a $\chi^{2}$ test or Fisher exact test for categorical variables and a $t$-test or Kruskal-Wallis test for continuous variables. We investigated the independent association between CAA pattern and outcomes (death or disability) with multivariable logistic regression analysis and adjusted for assigned treatment group, age, the presence of HTN, AF at baseline, and other covariates. These covariates were selected a prior based on the known predictors of these outcomes. The participants were followed up for 90 days and 12 months.

Separate univariable and multivariable ordinal regression analyses were used to assess the factors predicting of MoCA 


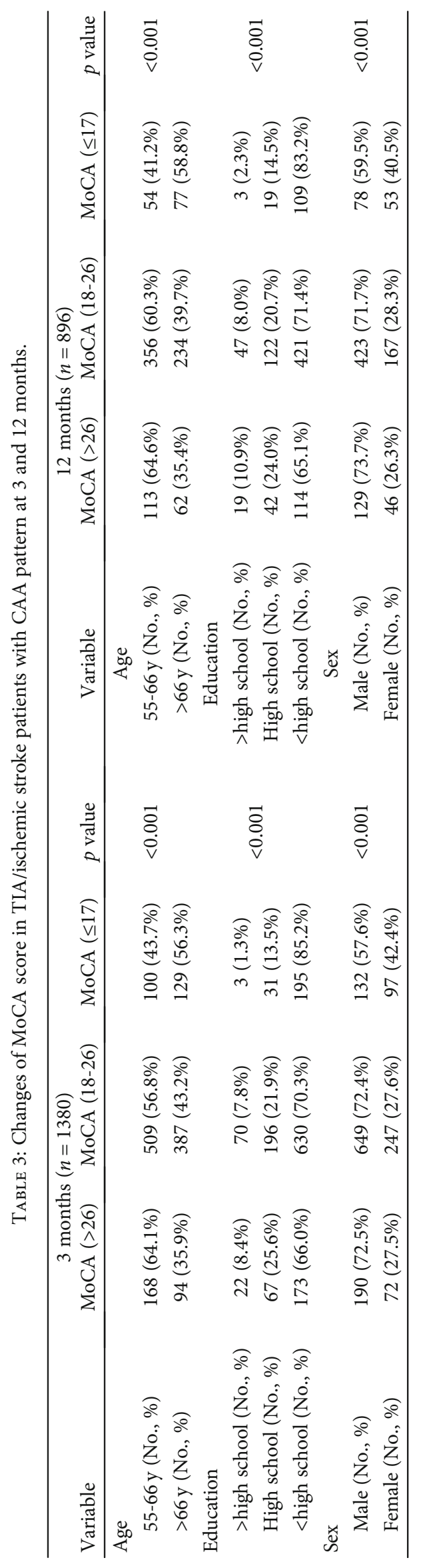


TABLE 4: Factors predicting of MoCA score improvement in TIA/ischemic stroke patients with CAA pattern at 3 and 12 months.

\begin{tabular}{|c|c|c|c|c|c|}
\hline \multicolumn{3}{|c|}{3 months } & \multicolumn{3}{|c|}{12 months } \\
\hline Variable & OR $(95 \% \mathrm{CI})$ & $p$ value & Variable & OR $(95 \% \mathrm{CI})$ & $p$ value \\
\hline Age $(>66 y)$ & $1.51(1.19-1.92)$ & 0.001 & Age $(>66 y)$ & $1.66(1.23-2.24)$ & 0.001 \\
\hline Education (high school) & $0.70(0.52-0.93)$ & 0.02 & Education (high school) & $0.80(0.56-1.16)$ & 0.24 \\
\hline Education (>high school) & $0.52(0.32-0.82)$ & 0.005 & Education (> high school) & $0.45(0.26-0.77)$ & 0.004 \\
\hline Sex (female) & $1.43(1.11-1.85)$ & 0.006 & & & \\
\hline
\end{tabular}

Education (>high school): college graduate or professional school.

in patients, multivariable analysis adjusting for age, HTN, $\mathrm{AF}$, prior ischemic stroke, alcohol, glucose, $3 \mathrm{~m} \mathrm{MoCA}$, and Fazekas scale.

All tests were 2 sided, and significance level was accepted at .05. All analyses were conducted using SAS version 9.4 (SAS Institute Inc., Cary, NC, USA).

2.7. Ethical Standards. The study received ethical approval by the ethics committee at Beijing Tiantan Hospital (No. KY2015-001-01). All patients or their legally authorized representative provided written informed consent.

\section{Results}

In August 2015-January 2018, 1620 of 2625 (61.71\%) participants were enrolled in this study. Neuroimaging evidence of probable/possible CAA in TIA or ischemic stroke was present in 291 patients (32.7\% female and mean [SD] age, 67.8). Table 1 provides characteristics of the comparing patients in CAA pattern with non-CAA pattern patients. Among 20 variables in this analysis, 7 variables were differed significantly (age, history of hypertension, AF, ischemic stroke, alcohol, glucose, and MoCA score at 3 months).

Eighty-two percent $(n=241)$ of 291 CAA patients had CMBs. 76 patients had superficial siderosis (cSS). 26 patients had both CMBs and cSS. Table 2 lists detailed neuroimaging findings in the CAA patients. Their median Fazekas scale score is 3.63 (IQR, 2-5). Compared to those without CAA, $\mathrm{CMBs}$ and cSS count in CAA patients were significant differences $(p<0.001$ and $p<0.001)$. Among the CAA patients with no more than five CMBs, CMBs were located in 156 patients (53.6\%) in the contex/grey-white junction, 47 patients $(16.2 \%)$ in the subcortical white matter, 6 patients $(2.1 \%)$ in the internal and external capsule, and 59 patients (20.3\%) in the cerebellum. The number of cases with cSS located in the frontal, parietal, temporal, occipital, centrum semiovale, and cerebellum was $10(3.4 \%), 14,(4.8 \%), 37$ (12.7\%), $13(4.5 \%), 6(2.1 \%)$, and $11(3.8 \%)$.

Age was divided into two groups: 55-66 years and more than 66 years. Education was divided into three groups: more than high school (college graduate or professional school), high school, and less than high school (junior school, primary school, and illiteracy). Sex was divided into two groups: female and male. Follow-up for 3 months and 12 months showed that there were significant differences in age, education level, and sex among different cognitive groups (Table 3). In multivariable ordinal logistic regression analyses of the predictors of cognitive function changes, the MoCA score of CAA pattern patients with a follow-up of 3-12 months was associated with age (more than 66 years), education (less than high school), and female sex. In multivariable analyses of predictors of cognitive function at 12 months, the MoCA score at 12-month follow-up was related to age (more than 66 years) and education (less than high school) (Table 4).

During the follow-up of 3 months, 30 of 286 (10.5\%) patients with CAA pattern had poor outcomes of death or disability (modified Rankin Scale score, 3-6), 24 of 244 (9.8\%) patients with CAA pattern had CMBs, and 12 of 93 (12.9\%) patients with CAA pattern had cSS. Patients with $\mathrm{CMB}$ count $>10$ were increased at risk of death or disability (adjusted relative risk [aRR 2.17; 95\% CI, 0.30-15.92]). In a multivariable analysis, patients with cSS were at increased risk of bad outcomes (adjusted relative risk [aRR 2.01; 95\% CI, 0.85-4.77]), especially the cSS count 1-2 (adjusted relative risk [aRR 2.07; 95\% CI, 0.88-4.90]), cSS topography including strictly lobar (adjusted relative risk [aRR 3.68; 95\% CI, 1.22-11.17]), and strictly deep (adjusted relative risk [aRR 2.19; 95\% CI, 0.59-17.35]) (Table 5).

37 of $281(13.2 \%)$ patients with CAA pattern had poor outcomes of death or disability at follow-up 12 months. The lack of association between CMBs and the outcomes of patients with CAA pattern was consistent with the present. In multivariable analyses of the outcomes followed up 12 months, the various of cSS consistent at 3 months, cSS topography mixed (adjusted relative risk [aRR 1.25; 95\% CI, 0.423.73]) were also associated with death or disability (Table 6).

\section{Discussion}

In this study, we have identified clinical and neuroimaging risk factor association with probable/possible CAA in TIA or ischemic stroke on admission. The increased risk with following characteristics: older age, history of hypertension, $\mathrm{AF}$ and ischemic stroke, alcohol, glucose on admission, and lower MoCA score at 3 months. More studies have been reported that the vascular factors such as age and history of hypertension are associated with of CAA $[5,6,16]$. Our findings suggested that we should pay more attention to the history of ischemic stroke and others. Among the non-CAA patients, the proportion with hypertension was surprisingly low (62.7\%). This may be explained that the non-CAA group with nonhypertensive ICHs is due to vascular malformations.

In the multivariate analyses, age is a risk factor associated with CAA pattern. In addition, age is also a strong predictor 
TABLE 5: Outcomes of CAA pattern in TIA/ischemic stroke patients at 3 months.

\begin{tabular}{|c|c|c|c|}
\hline \multirow[b]{2}{*}{ Outcomes } & \multicolumn{3}{|c|}{ Death or disability (mRS 3-6) } \\
\hline & $\begin{array}{l}\text { No. of } \\
\text { events }\end{array}$ & $\begin{array}{c}\text { Crude RR }(95 \% \\
\text { CI) }\end{array}$ & $\begin{array}{l}\text { Adjusted RR } \\
\text { (95\% CI) }\end{array}$ \\
\hline \multicolumn{4}{|l|}{ CMB status $^{\mathrm{a}}$} \\
\hline No CMB $(n=42)$ & $6 / 42$ & 1 (reference) & 1 (reference) \\
\hline $\mathrm{CMB}(n=244)$ & $24 / 244$ & $0.66(0.25-1.71)$ & $0.33(0.11-0.98)$ \\
\hline \multicolumn{4}{|l|}{$\mathrm{CMB}$ count $^{\mathrm{a}}$} \\
\hline None $(n=42)$ & $6 / 42$ & 1 (reference) & 1 (reference) \\
\hline $1-2(n=165)$ & $13 / 165$ & $0.51(0.18-1.44)$ & $0.27(0.08-0.86)$ \\
\hline $3-10(n=72)$ & $9 / 72$ & $0.86(0.28-2.60)$ & $0.36(0.10-1.31)$ \\
\hline$>10(n=7)$ & $2 / 7$ & $2.40(0.38-15.33)$ & $2.17(0.30-15.92)$ \\
\hline \multicolumn{4}{|l|}{ CMB topography ${ }^{a}$} \\
\hline None $(n=67)$ & $8 / 67$ & 1 (reference) & 1 (reference) \\
\hline $\begin{array}{l}\text { Strictly lobar } \\
(n=133)\end{array}$ & $11 / 133$ & $0.67(0.25-1.74)$ & $0.50(0.17-1.46)$ \\
\hline $\begin{array}{l}\text { Strictly deep } \\
(n=10)\end{array}$ & $1 / 10$ & $0.82(0.09-7.35)$ & $0.57(0.06-5.73)$ \\
\hline Mixed $(n=76)$ & $10 / 76$ & $1.12(0.41-3.02)$ & $0.70(0.22-2.18)$ \\
\hline \multicolumn{4}{|l|}{ cSS status $^{\mathrm{b}}$} \\
\hline No cSS $(n=192)$ & $18 / 192$ & 1 (reference) & 1 (reference) \\
\hline $\operatorname{cSS}(n=93)$ & $12 / 93$ & $1.43(0.66-3.11)$ & $2.01(0.85-4.77)$ \\
\hline \multicolumn{4}{|l|}{$\mathrm{cSS}_{\text {count }}{ }^{\mathrm{b}}$} \\
\hline None $(n=192)$ & $18 / 192$ & 1 (reference) & 1 (reference) \\
\hline $1-2(n=89)$ & $12 / 89$ & $1.51(0.69-3.28)$ & $2.07(0.88-4.90)$ \\
\hline $3-10(n=4)$ & $0 / 4$ & - & - \\
\hline$>10(n=0)$ & 0 & 0 & 0 \\
\hline \multicolumn{4}{|l|}{ cSS topography ${ }^{b}$} \\
\hline None $(n=192)$ & $18 / 192$ & 1 (reference) & 1 (reference) \\
\hline Strictly lobar $(n=35)$ & $6 / 35$ & $2.00(0.73-5.46)$ & $3.68(1.22-11.17)$ \\
\hline Strictly & $2 / 10$ & $2.42(0.48-12.26)$ & $2.19(0.59-17.35)$ \\
\hline Mixed $(n=48)$ & $4 / 48$ & $0.88(0.28-2.73)$ & $0.92(0.25-3.47)$ \\
\hline
\end{tabular}

Abbreviations: RR: relative risk; CMB: cerebral microbleed; cSS: cortical superficial siderosis. Multivariable analysis adjusted for age, HTN (history of hypertension), AF (history of atrial fibrillation), prior ischemic stroke, alcohol, glucose, $3 \mathrm{~m} \mathrm{MoCA}$, and Fazekas scale. ${ }^{\mathrm{a}}$ Data were missing for 5 patients. ${ }^{\mathrm{b}}$ Data were missing for 6 patients.

factor of development of cognitive impairment in the first 1 year after diagnosis of CAA pattern in TIA/ischemic stroke. The increase with age is well known as the prevalence of cognitive impairment in Alzheimer's disease and other neurodegenerative diseases $[17,18]$. The MoCA score would be higher in patients who improve between 3 and 12 months with lower age, female sex, and higher education. It might be interesting that they did contribute to prediction of cognitive decline would be recovering in patients with CAA pattern. Though sex may not be a strong predictive factor with MoCA at 12-month follow-up, but the female sex patients may still recover better at 3 months and have higher scores at 12 months. With higher level education, patients should get more better managements, more preventive interventions, and appropriate treatment to improve cognitive performance, as previously been reported in fewer years [19].
TABLE 6: Outcomes of CAA pattern in TIA/ischemic stroke patients at 12 months.

\begin{tabular}{|c|c|c|c|}
\hline \multirow[b]{2}{*}{ Outcomes } & \multicolumn{3}{|c|}{ Death or disability (mRS 3-6) } \\
\hline & $\begin{array}{l}\text { No. of } \\
\text { events }\end{array}$ & $\begin{array}{c}\text { Crude RR }(95 \% \\
\text { CI) }\end{array}$ & $\begin{array}{l}\text { Adjusted RR } \\
\text { (95\% CI) }\end{array}$ \\
\hline \multicolumn{4}{|l|}{ CMB status ${ }^{\mathrm{a}}$} \\
\hline No CMB $(n=42)$ & $8 / 42$ & 1 (reference) & 1 (reference) \\
\hline $\mathrm{CMB}(n=239)$ & $29 / 239$ & $0.59(0.25-1.39)$ & $0.26(0.09-0.71)$ \\
\hline \multicolumn{4}{|l|}{$\mathrm{CMB}$ count $^{\mathrm{a}}$} \\
\hline None $(n=42)$ & $8 / 42$ & 1 (reference) & 1 (reference) \\
\hline $1-2(n=162)$ & $16 / 162$ & $0.47(0.18-1.18)$ & $0.22(0.08-0.65)$ \\
\hline $3-10(n=70)$ & $12 / 70$ & $0.88(0.33-2.37)$ & $0.34(0.10-1.10)$ \\
\hline$>10(n=7)$ & $1 / 7$ & $0.71(0.07-6.74)$ & $0.46(0.04-4.76)$ \\
\hline \multicolumn{4}{|l|}{ CMB topography } \\
\hline None $(n=68)$ & $11 / 68$ & 1 (reference) & 1 (reference) \\
\hline $\begin{array}{l}\text { Strictly lobar } \\
(n=128)\end{array}$ & $12 / 128$ & $0.54(0.22-1.29)$ & $0.36(0.14-0.96)$ \\
\hline Strictly deep $(n=10)$ & $2 / 10$ & $1.30(0.24-6.94)$ & $0.94(0.15-5.75)$ \\
\hline Mixed $(n=75)$ & $12 / 75$ & $0.99(0.40-2.41)$ & $0.54(0.19-1.53)$ \\
\hline \multicolumn{4}{|l|}{ cSS status $^{\mathrm{b}}$} \\
\hline No cSS $(n=188)$ & $24 / 188$ & 1 (reference) & 1 (reference) \\
\hline $\operatorname{cSS}(n=92)$ & $13 / 92$ & $1.12(0.54-2.33)$ & $1.63(0.73-3.67)$ \\
\hline \multicolumn{4}{|l|}{$\mathrm{cSS}_{\text {count }}{ }^{\mathrm{b}}$} \\
\hline None $(n=188)$ & $24 / 188$ & 1 (reference) & 1 (reference) \\
\hline $1-2(n=89)$ & $13 / 89$ & $1.17(0.57-2.42)$ & $1.66(0.74-3.73)$ \\
\hline $3-10(n=3)$ & $0 / 3$ & - & - \\
\hline$>10(n=0)$ & 0 & 0 & 0 \\
\hline \multicolumn{4}{|l|}{ cSS topography ${ }^{\mathrm{b}}$} \\
\hline None $(n=188)$ & $24 / 188$ & 1 (reference) & 1 (reference) \\
\hline $\begin{array}{l}\text { Strictly lobar } \\
(n=35)\end{array}$ & $5 / 35$ & $1.14(0.40-3.22)$ & $2.05(0.67-6.29)$ \\
\hline $\begin{array}{l}\text { Strictly deep } \\
(n=10)\end{array}$ & $2 / 10$ & $1.71(0.34-8.53)$ & $2.09(0.39-11.18)$ \\
\hline Mixed $(n=47)$ & $6 / 47$ & $1.00(0.38-2.61)$ & $1.25(0.42-3.73)$ \\
\hline
\end{tabular}

Abbreviations: RR: relative risk; CMB: cerebral microbleed; cSS: cortical superficial siderosis. Multivariable analysis adjusted for age, HTN (history of hypertension), AF (history of atrial fibrillation), prior ischemic stroke, alcohol, glucose, $3 \mathrm{~m} \mathrm{MoCA}$, and Fazekas scale. ${ }^{\mathrm{a}}$ Data were missing for 10 patients. ${ }^{\mathrm{b}}$ Data were missing for 11 patients.

In this cohort study, CAA pattern was associated with the presence of WMHs. We observed greater death or disability at 3 and 12 months in the patients with cSS, compared with those with $\mathrm{CMBs}$, except $\mathrm{CMB}$ counts $>10$. It seems that a strong predictor of outcomes of CAA pattern was associated with cSS count and topography, irrespective of whether the outcomes at 3 months or 1 year. Some studies observed that cSS affected both the supra- and the infratentorial compartments associated with CAA patients [20].

4.1. Strengths and Limitations. Our study has some limitations. Though we analyzed the imaging markers including $\mathrm{WMH}, \mathrm{CMBs}$, and cSS, we did not collect other neuroimaging various assessments for the outcomes of death or 
disability at 3 and 12 months. The cSS sample size was smaller than expected to get more information. It seems to limit us to correctly assess factors though cSS count and topography. Moreover, this study only included a follow-up of the first one year after diagnosis, and few patients with cognitive clinical symptoms developed or recovered slowly. Our findings should therefore be considered in the context of predicting cognitive impairment with longer follow-up. We should further validate the factors and the predicting risks in other cohorts and explain further in the appropriate clinical context.

\section{Conclusions}

In this study, age, history of hypertension, and ischemic stroke were strong factors associated with CAA pattern, whereas higher educational level, female sex, and young age maybe recover the cognitive impairment. cSS was significant for outcomes. These findings may provide some support of the outcomes of the CAA pattern in TIA/ischemic stroke.

\section{Data Availability}

In this study, we used data from China National Clinical Research Center for Neurological Diseases to mimic the design and to examine the China National Stroke Registry III Cognitive Subgroup (ICONS). The data is available on request to China National Clinical Research Center for Neurological Diseases.

\section{Disclosure}

The funding source had no role in the design and conduct of the study; collection, management, analysis, and interpretation of the data; preparation, review, or approval of the manuscript; and the decision to submit the manuscript for publication.

\section{Conflicts of Interest}

The authors declare that they have no conflicts of interest.

\section{Authors' Contributions}

Drs. Zhang Q and Wang Y had full access to all the data in the study and took responsibility for the integrity of the data and the accuracy of the data analysis. Study concept and design was conducted by Zhang Q and Wang Y. Acquisition, analysis, or interpretation of data was done by all authors. Drafting of the manuscripts was carried out by Zhang Q. Critical revision of the manuscript for important intellectual content was performed by Zhang Q and Wang Y. Intellectual content was done by all authors. Statistical analysis was conducted by Wang $A$ and Zhang Y. Obtained funding was done by Wang Y and Zhang Q. Administrative, technical, or material support was carried out by Meng X and Wang Y. Study supervision was done by Zhang Q and Wang Y.

\section{Acknowledgments}

This study is carried out as a collaborative study supported by the Ministry of Science and Technology of the People's Republic of China (Grant Nos. 2016YFC0901002, 2017YFC1310901, 2017YFC1310902, and 2018YFC1312300), National Science and Technology Major Project (2017ZX09304018), and Beijing Municipal Science \& Technology Commission (Grant No. D171100003017002).

\section{References}

[1] S. M. Greenberg, B. J. Bacskai, M. Hernandez-Guillamon, J. Pruzin, R. Sperling, and S. J. van Veluw, "Cerebral amyloid angiopathy and Alzheimer disease-one peptide, two pathways," Nature Reviews. Neurology, vol. 16, no. 1, pp. 30-42, 2020.

[2] E. E. Smith and M. B. Maas, "To predict recurrence in cerebral amyloid angiopathy, look to the subarachnoid space," Neurology, vol. 94, no. 9, pp. 375-376, 2020.

[3] A. R. Switzer, I. Cheema, C. R. McCreary et al., "Cerebrovascular reactivity in cerebral amyloid angiopathy, Alzheimer disease, and mild cognitive impairment," Neurology, vol. 95, no. 10, pp. e1333-e1340, 2020.

[4] M. Kivipelto, E. L. Helkala, M. P. Laakso et al., "Midlife vascular risk factors and Alzheimer's disease in later life: longitudinal, population based study," BMJ, vol. 322, no. 7300, pp. 1447-1451, 2001.

[5] R. F. Gottesman, A. L. C. Schneider, Y. Zhou et al., "Association between midlife vascular risk factors and estimated brain amyloid deposition," Journal of the American Medical Association, vol. 317, no. 14, pp. 1443-1450, 2017.

[6] A. Charidimou, S. Martinez-Ramirez, Y. D. Reijmer et al., "Total magnetic resonance imaging burden of small vessel disease in cerebral amyloid angiopathy: an imaging-pathologic study of concept validation," JAMA Neurology, vol. 73, no. 8, pp. 994-1001, 2016.

[7] S. M. Greenberg, R. A.-S. Salman, G. J. Biessels et al., "Outcome markers for clinical trials in cerebral amyloid angiopathy," Lancet Neurology, vol. 13, no. 4, pp. 419-428, 2014.

[8] G. Banerjee, The CROMIS-2 collaborators, E. Chan et al., "Effect of small-vessel disease on cognitive trajectory after atrial fibrillation-related ischaemic stroke or TIA," Journal of Neurology, vol. 266, no. 5, pp. 1250-1259, 2019.

[9] S. M. Greenberg and A. Charidimou, "Diagnosis of cerebral amyloid angiopathy: evolution of the Boston criteria," Stroke, vol. 49, no. 2, pp. 491-497, 2018.

[10] Y. Wang, X. Liao, C. Wang et al., "Impairment of cognition and sleep after acute ischaemic stroke or transient ischaemic attack in Chinese patients: design, rationale and baseline patient characteristics of a nationwide multicentre prospective registry," Stroke and Vascular Neurology, vol. 6, no. 1, 2021.

[11] S. E. Kasner, "Clinical interpretation and use of stroke scales," Lancet Neurology, vol. 5, no. 7, pp. 603-612, 2006.

[12] Z. S. Nasreddine, N. A. Phillips, V. Ã. C. BÃCdirian et al., "Mild cognitive impairment and its management in older people," Journal of the American Geriatrics Society, vol. 53, no. 4, pp. 695-699, 2005.

[13] C. Cordonnier, G. M. Potter, C. A. Jackson et al., "Improving interrater agreement about brain microbleeds: development 
of the Brain Observer Micro Bleed Scale (BOMBS)," Stroke, vol. 40, no. 1, pp. 94-99, 2009.

[14] A. Shoamanesh, A. Morotti, J. M. Romero et al., "Cerebral microbleeds and the effect of intensive blood pressure reduction on hematoma expansion and functional outcomes: a secondary analysis of the ATACH-2 randomized clinical trial," JAMA Neurology, vol. 75, no. 7, pp. 850-859, 2018.

[15] A. Shoamanesh, L. A. Pearce, C. Bazan et al., "Microbleeds in the Secondary Prevention of Small Subcortical Strokes Trial: stroke, mortality, and treatment interactions," Annals of Neurology, vol. 82, no. 2, pp. 196-207, 2017.

[16] R. F. Gottesman, M. S. Albert, A. Alonso et al., "Associations between midlife vascular risk factors and 25-year incident dementia in the Atherosclerosis Risk in Communities (ARIC) cohort," JAMA Neurology, vol. 74, no. 10, pp. 1246-1254, 2017.

[17] Z. Fridaus and T. D. Singh, "An insight in pathophysiological mechanism of Alzheimer's disease and its management using plant natural products," Mini-Reviews in Medicinal Chemistry, vol. 21, no. 1, pp. 35-57, 2021.

[18] A. Schrag, U. F. Siddiqui, Z. Anastasiou, D. Weintraub, and J. M. Schott, "Clinical variables and biomarkers in prediction of cognitive impairment in patients with newly diagnosed Parkinson's disease: a cohort study," Lancet Neurology, vol. 16, no. 1, pp. 66-75, 2017.

[19] S. A. Eshkoor, C. Y. Mun, C. K. Ng, and T. A. Hamid, "Mild cognitive impairment and its management in older people," Clinical Interventions in Aging, vol. 10, pp. 687-693, 2015.

[20] J. Linn, A. Halpin, P. Demaerel et al., "Prevalence of superficial siderosis in patients with cerebral amyloid angiopathy," $\mathrm{Neu}$ rology, vol. 74, no. 17, pp. 1346-1350, 2010. 